\title{
Master of Business Administration
}

National Cancer Institute

\section{Source}

National Cancer Institute. Master of Business Administration. NCI Thesaurus. Code C39449.

Master of Business Administration is a master's degree in business. 